\title{
Epidemiological Models and Lyapunov Functions
}

\author{
A. Fall ${ }^{a, c}$, A. Iggidr $^{a}$, G. Sallet ${ }^{a 1}$ and J.J. Tewa ${ }^{a, b}$ \\ ${ }^{a}$ INRIA Lorraine \& Université Paul Verlaine, Metz LMAM (UMR CNRS 7122) \\ I.S.G.M.P. Bât A, Ile du Saulcy, 57045 Metz Cedex 01, France \\ ${ }^{b}$ Université de Yaoundé, Cameroun \\ ${ }^{c}$ Université de Saint-Louis, Sénégal
}

\begin{abstract}
We give a survey of results on global stability for deterministic compartmental epidemiological models. Using Lyapunov techniques we revisit a classical result, and give a simple proof. By the same methods we also give a new result on differential susceptibility and infectivity models with mass action and an arbitrary number of compartments. These models encompass the so-called differential infectivity and staged progression models. In the two cases we prove that if the basic reproduction ratio $\mathcal{R}_{0} \leq 1$, then the disease free equilibrium is globally asymptotically stable. If $\mathcal{R}_{0}>1$, there exists an unique endemic equilibrium which is asymptotically stable on the positive orthant.
\end{abstract}

Key words: nonlinear dynamical systems, global stability, Lyapunov methods, differential susceptibility models

AMS subject classification: 34A34, 34D23, 34D40, 92D30

\section{Introduction}

The primary objective of this paper is to give two results on global stability for some epidemiological models using Lyapunov techniques. Using a new result for systems of the type $\dot{x}=A(x) x$, we revisit a celebrated result of Lajmanovitch and Yorke [39] and give a simple proof. The second result is for differential susceptibility and infectivity models with mass action. We generalize a result of $[28,24]$. But before we will give an overview of the literature concerning the problem of stability in epidemiological models

\footnotetext{
${ }^{1}$ Corresponding author. E-mail: sallet@ loria.fr
} 
In [59] J.A. Jacquez says :

A major project in deterministic epidemiological modeling of heterogeneous populations is to find conditions for local and global stability of the equilibria and to work out the relations among these stability conditions, the threshold of epidemic take-off, and endemicity, and the basic reproduction.

We denote in this paper by $\mathcal{R}_{0}$ the basic reproduction number. The basic reproduction number, a key concept in epidemiology, is defined as the expected number of new cases of infection caused by a typical infected individual in a population of susceptibles only and is an ingredient in almost all papers using mathematical modeling for infectious diseases. An abundant literature have been devoted to $\mathcal{R}_{0}$ (see $[8,10,67,17,18,34,59,66]$ and the references therein) after the seminal paper [9].

The citation before was written in 1982, at this time no too many results was known. What is the situation 25 year later? As early as 1976 the stability analysis for the classic SIR or SIRS models was well known [20,19]. The reason was that the study of stability for these models was reduced to the study of 2-dimensional systems, hence phase methods could be used : PoincaréBendixson theorem. Periodic orbits are ruled out using Dulac criteria or condition of Busenberg and van den Driessche [7].

For many infectious diseases the transmission occurs in a heterogeneous population, so the epidemiological model must divide the population into subpopulations or groups, in which the members have similar characteristics. This division into groups can be based not only on mode of transmission, contact patterns, latent period, infectious period, genetic susceptibility or resistance, and amount of vaccination or chemotherapy, but also on social, cultural, economic, demographic, or geographic factors. This is the rationale for the introduction of multi-group models. In the epidemiological literature, the term "multi-group" usually refers to the division of a heterogeneous population into several homogeneous groups based on individual behaviour. The interest in multigroup endemic models origina lly stems from sexual transmitted diseases such as gonorrhea or HIV/AIDS. The pioneering paper of Lajmanovitch and Yorke in 1976 [39] provides a complete description of the dynamics of $n$ groups of SIS systems for subpopulations of constant size. The authors use Lyapunov techniques to prove that either all trajectories in $\mathbb{R}_{+}^{n}$ tends to 0 , or else there is a unique endemic equilibrium $\bar{x}$ in the positive orthant and trajectories in $\mathbb{R}_{+}^{n} \backslash\{0\}$ tends to $\bar{x}$.

Other types of high dimensional systems are the so-called differential infectivity (DI) and staged progression (SP) models. The staged progression model $[59,29,34]$ has a single uninfected compartment, and infected individuals progress through several stages of the disease with changing infectivity. This model is applicable to disea se with changing infectivity during the infectious period such that HIV or disease with asymptomatic carriers such that HBV or tuberculosis. The differential infectivity model has been also introduced to take into account some specificity of HIV/AIDS. In a DI model the infected population is subdivided into subgroups of different infectivity. Upon infection, an individual enters some subgroup with a certain probability and stays in this subgroup until becoming inactive in transmission.

For multigroup SEIRS models of constant size many results have demonstrated the global stability of the disease free equilibrium when $\mathcal{R}_{0}<1$ and the local asymptotic stability of an 
unique endemic equilibrium when $\mathcal{R}_{0}>1[21,64,65]$. The most difficult task is the global stability of the endemic equilibrium when $\mathcal{R}_{0}>1$, when this is possible. Actually for general multigroup models the uniqueness of the endemic equilibrium non longer holds and the disease free equilibrium may be locally, but not globally, asymptotically stable [67, 34, 59].

The global stability when $\mathcal{R}_{0}>1$ of SEIR models with constant size has long been conjectured but only proven in 1995 [43]. The proof relies heavily on the competitive structure of the system, and the fact that 3 dimensional competitive systems satisfy the Poincaré-Bendixson theorem [22, $61,60]$. When the system is not competitive another approach consists to show that the system satisfies a Bendixson criterion which is robust under $\mathcal{C}^{1}$ perturbation $[44,62,41,48]$.

Since these path-breaking papers numerous results of global stability for the endemic equilibrium have been obtained for low dimensional systems;

- For SEIRS systems, with fraction of classes (then the system reduces to a 3-dimensional systems) for small or large temporary immunity [45],

- For SEIR model with vertical transmission [46]. The study of stability is reduced to a 3dimensional system. Since this system is not competitive the second approach is used,

- For SEIR model with varying population size [42] for the system of fraction of classes,

- For SIRV models (V for vaccinate class) with constant population size and mild parameters constraints [2],

- For SVEIR models [14] with small mortality,

- For Staged progression models in dimension 3 and 4 [53],

- For SEI models with immigration of latent and infectious [54],

- A model of dengue which is reduced to a 3 dimensional competitive system [67],

- A 5 dimensional staged progression model [13], for which the asymptotic stability of the endemic equilibrium reduces to a 3 dimensional system permitting to apply the Li-Muldowney technique [44].

For arbitrary dimensional system, the most promising method may be that of Lyapunov. The systematic use of Lyapunov function in studying stability problems is relatively recent. The exception is the result of Lajmanovitch and Yorke evoked before. However, Lasalle-Lyapunov theory has been used in $[50,57,34,59]$ to study the stability of classic SIRS models.

In 2004, Korobeinikov and Maini using a Lyapunov function [36] demonstrate simply the result of $\mathrm{Li}$ and Muldowney for the endemic equilibrium. The Lyapunov function used is $V=$ $\sum_{i=1}^{n} a_{i}\left(x_{i}-\bar{x} \ln x_{i}\right)$. This function has a long history of application to Lotka-Volterra models $[5,12,16,63]$ and was originally discovered by Volterra himself, although he did not use the 
vocabulary and the theory of Lyapunov functions. Since epidemic models are "Lotka-Volterra" like models, the pertinence of this function is not surprising. This Volterra-Lyapunov function has been used in epidemiological models at the end of the eighties.

Beretta and Capasso [4] use a skew-symmetry condition on the Jacobian of the matrix of the system to give a necessary condition for the global stability of the endemic equilibrium.

For a SIRS multigroup model with constant subgroup sizes, Lin and So [49] show that the endemic equilibrium is globally asymptotically stable if the contact rate between subgroups is small. These two results are actually perturbation results of the situation where the endemic equilibrium is known to be globally stable.

Since the publication of the result of Korobeinikov and Maini the "Volterra-like" Lyapunov functions has been used to address the stability of high-dimensional systems with mass action. The difficulty is in choosing the coefficient and in proving that the derivative is nonnegative. The global stability of DI model with mass action is demonstrated in [52]. The global stability of SP model, eventually with latent classes, with mass action is proved in [15, 32]. Stability of intra-host models with different strains [30] (which contained as a particular case, $S E_{1} \cdots E_{k} I R$ models with multiple strains) is treated in $[1,30]$. The stability of differential and staged progression latent classes, with one infectious class is solved in [32]. Two models of tuberculosis are studied in [55]. The stability of a model with complex graph interaction between latent classes and one infectious class is addressed in [56].

We give a brief outline of the paper. In Section 2, we consider a system similar to the system in [39]. We compute $\mathcal{R}_{0}$ and prove that if $\mathcal{R}_{0} \leq 1$, the DFE is globally asymptotically stable and if $\mathcal{R}_{0}>1$, then a unique equilibrium exists which is globally asymptotically stable on $\mathbb{R}_{+}^{n} \backslash\{0\}$. In Section 3, we present a system with different classes of susceptible individuals and staged progression through latency and infectious classes. Using a "Volterra-like" Lyapunov function we obtain results as before : if $\mathcal{R}_{0} \leq 1$ the DFE is globally asymptotically stable and if $\mathcal{R}_{0}>1$ then a unique equilibrium exists which is globally asymptotically stable on the positive orthant.

\section{A $n$ groups SIS model}

Throughout the paper we will use the following classical notations. We identify vectors of $\mathbb{R}^{n}$ with $n \times 1$ column vectors. The Euclidean inner product is denoted by $\langle\mid\rangle$, then $\|z\|_{2}^{2}=\langle z \mid z\rangle$ is the usual Euclidean norm. The family $\left\{e_{1}, \cdots, e_{n}\right\}$ denotes the canonical basis of the vector space $\mathbb{R}^{n}$. We denote by 1 the vector with all components equal to 1 , i.e. $\mathbf{1}=e_{1}+\cdots+e_{n}$.

If $x \in \mathbb{R}^{n}$ we denote by $x_{i}$ the $i$-th component of $x$. Equivalently $x_{i}=\left\langle x \mid e_{i}\right\rangle$. For a matrix $A$ we denote by $A(i, j)$ the entry in row $i$, column $j$. For matrices $A, B$ we write $A \leq B$ if $A(i, j) \leq B(i, j)$ for all $i$ and $j, A<B$ if $A \leq B$ and $A \neq B, A \ll B$ if $A(i, j)<B(i, j)$ for all $i$ and $j$. The notation $A^{T}$ denotes the transpose of $A$. Then $\left\langle v_{1} \mid v_{2}\right\rangle=v_{1}^{T} v_{2}$. The notation $A^{-T}$ will denote the transpose of the inverse of $A$. If $x \in \mathbb{R}^{n}$, we denote by $\operatorname{diag}(x)$ the diagonal matrix whose diagonal elements are given by $x$.

A Metzler matrix $A$ is a matrix such that $A(i, j) \geq 0$ for any indices $i \neq j[6,33,51]$. These matrices are also called quasipositive matrices [61]. Metzler matrices are the opposite of $M$ - 
matrices $[6,67]$. We prefer to use Metzler matrices since they appear naturally in compartmental systems.

In this section we will consider the following system

$$
\dot{x}=[D+B-\operatorname{diag}(x) B] x .
$$

where $D$ is a stable Metzler matrix and $B \geq 0$ is a nonnegative irreducible matrix.

To motivate the consideration of such a system we consider $n$ groups with constant population size and a disease which confer no immunity after recovery. We model the contact by the mass action law. If we denote by $S_{i}$ and $I_{i}$ the respective number of susceptible and infectious individuals in group $i, N_{i}=S_{i}+I_{i}$, the system is, for $i=1, \cdots, n$

$$
\left\{\begin{aligned}
\dot{S}_{i} & =\mu_{i} N_{i}-\mu_{i} S_{i}-\sum_{j=1}^{n} \beta_{i, j} \frac{S_{i}}{N_{i}} I_{i}+\gamma_{i} I_{i} \\
\dot{I} & =\sum_{j=1}^{n} \beta_{i, j} \frac{S_{i}}{N_{i}} I_{i}-\left(\gamma_{i}+\mu_{i}\right) I_{i} .
\end{aligned}\right.
$$

Since the population is constant, it is sufficient to know the $I_{i}$. If we set $x_{i}=\frac{I_{i}}{N_{i}}, \tilde{\beta}_{i, j}=\beta_{i, j} N_{j}$ and $\alpha_{i}=\gamma_{i}+\mu_{i}$ we obtain a system of ODE

$$
\dot{x}_{i}=\left(1-x_{i}\right) \sum \tilde{\beta}_{i, j} x_{j}-\alpha_{i} x_{i} .
$$

that we can write in compact form

$$
\dot{x}=[D+B-\operatorname{diag}(x) B] x .
$$

with $B=\left(\tilde{\beta}_{i, j}\right)$ and $D=-\operatorname{diag}\left(\alpha_{i}\right)$. This system is the system considered in [39], where the system addressed has the structure of (2.1). In this model, the matrix $B$ describes the contact interaction between groups. We recall the following definition $[6,61,66]$.

Definition 2..1. A matrix A of size $n \times n, n \geq 2$ is called irreducible if for any proper subset I of $\{1, \cdots, n\}$ there are $i \in I$ and $j \notin I$ such that $A(i, j) \neq 0$.

Epidemiogically speaking the irreducibility of $B$ (or $Q$ ) means that no group is contact isolated in and out from the remaining groups. It is now easy to interpret the meaning of the system (2.1). The matrix $D$ describes the transfer of individuals out of compartments and $B-\operatorname{diag}(x) B$ the disease transmission. The model can also be written $\dot{x}=[D+\operatorname{diag}(1-x) B] x$. It is clear that $[0,1]^{n}$ is a compact positively invariant absorbing set for this system.

\subsection{The basic reproduction number}

We denote by $\rho(A)$ the spectral radius of a matrix $A$, which is defined, if $\operatorname{Sp}(A)$ denotes the spectrum of $A$, by

$$
\rho(A)=\max \{|\lambda| \mid \lambda \in(\operatorname{Sp}(A)\}
$$


and the stability modulus $\alpha(A)$

$$
\alpha(A)=\max \{\Re(\lambda) \mid \lambda \in(S p(A)\} .
$$

Using the framework of [67] the matrix $-D^{-1} B$ is the next generation matrix of (2.1) and the basic reproduction number is $\mathcal{R}_{0}=\rho\left(-D^{-1} B\right)$. We now will use a result of Varga $[69,68]$ (rewritten in term of Metzler matrices)

Definition $2 . .2$ (Regular splitting). : For a real Metzler matrix $\mathrm{M}, \mathbf{M}=\boldsymbol{\Lambda}+\mathbf{N}$ is a regular splitting if $\Lambda$ is a Metzler stable matrix and $\mathbf{N} \geq 0$ is a nonnegative matrix.

Now we can give the following classical theorem.

Proposition 2..1 (Varga, 1962, Theorem 3.13, [69]). Let $\mathbf{M}=\boldsymbol{\Lambda}+\mathbf{N}$ be a regular splitting of $\mathbf{M}$, a real Metzler matrix. Then $\mathbf{M}$ is Metzler stable if and only if $\rho\left(-\mathbf{N} \Lambda^{-1}\right)<1$.

The proof of Proposition 2..1 is in Varga (1960). It is also in Bermann and Plemmons [6]: the condition $N_{45}$ expressed in terms of $M$-matrices. We see from this proposition, by a continuity argument, that for any regular splittings of a Metzler matrix $\mathbf{M}$ we have

$$
\begin{aligned}
& \alpha(\mathbf{M})<0 \Longleftrightarrow \rho\left(-\mathbf{N} \boldsymbol{\Lambda}^{-1}\right)<1, \\
& \alpha(\mathbf{M})=0 \Longleftrightarrow \rho\left(-\mathbf{N} \boldsymbol{\Lambda}^{-1}\right)=1 .
\end{aligned}
$$

Thus any regular splitting gives an equivalent threshold condition $\alpha(M)$ on the parameters. This has a consequence for our system : $D+B$ is a regular splitting and the stability of $D+B$ is completely related to $\mathcal{R}_{0}$ and its position relatively to 1 . Since this equivalence is independent from the splitting, we can replace the system (2.1) by the same system where we assume that $D$ is a diagonal matrix and incorporating the off-diagonal elements in $B$, this modification let the new matrix $B$ still irreducible. This does not change the generality of the conclusion. However only the original $\rho\left(-D^{-1} B\right)$ has a biological meaning, the others are equivalent thresholds. From now on we will assume that $D$ is a diagonal matrix.

\subsection{Existence and uniqueness of an endemic equilibrium}

We will show that there exists a unique equilibrium $\bar{x} \gg 0$ if and only if $\mathcal{R}_{0}>1$. An equilibrium such that $\bar{x} \gg 0$ is called a strongly endemic equilibrium. The method of proof is inspired by the methods used by Thieme $[21,66,64]$. We show that if there exists an endemic equilibrium $\bar{x}>0$ then $\mathcal{R}_{0}>1$. For the convenience of the reader we recall the following result on Metzler matrices [6]

Theorem 2..1. Let A be an irreducible Metzler matrix

1. If there exists $x>0$ such that $A x>\lambda x$ then $\alpha(A)>\lambda$. 
2. If there exists $x>0$ such that $\mu x>A x$ then $\mu>\alpha(A)$.

If $A$ is only Metzler, the preceding relations hold with $>$ replaced by $\geq$. If $A$ is an irreducible nonnegative matrix, we have analogous inequalities, obtained in replacing the stability modulus $\alpha(A)$ by the spectral radius $\rho(A)$ in the preceding inequalities.

If there exists an endemic equilibrium $\bar{x}>0$ it satisfies

$$
\bar{x}=-D^{-1} B \bar{x}+\operatorname{diag}(\bar{x}) D^{-1} B \bar{x} .
$$

Since $D$ is a Metzler matrix, then $-D^{-1}>0$ [61]. As $B$ is irreducible and $-D$ diagonal, with positive diagonal terms, $-D^{-1} B$ is also irreducible. Therefore $-D^{-1} B \bar{x} \gg 0$ and from the preceding relation, we deduce $\bar{x} \gg 0$. A consequence is also $\operatorname{diag}(\bar{x}) D^{-1} B \bar{x} \ll 0$. Finally we obtain

$$
\bar{x}<-D^{-1} B \bar{x} .
$$

which in turn implies using Theorem 2..1

$$
\mathcal{R}_{0}=\rho\left(-D^{-1} B\right)>1 \text {. }
$$

Conversely, we have to show that if $\mathcal{R}_{0}>1$, then there exists a unique strongly endemic equilibrium. An equilibrium satisfies

$$
(D+B) \bar{x}=\operatorname{diag}(\bar{x}) B \bar{x},
$$

equivalently,

$$
\bar{x}+\operatorname{diag}(\bar{x})\left(-D^{-1} B \bar{x}\right)=\bar{x}+\operatorname{diag}\left(-D^{-1} B \bar{x}\right) \bar{x}=-D^{-1} B \bar{x},
$$

which can be written

$$
\left[I+\operatorname{diag}\left(-D^{-1} B \bar{x}\right)\right] \bar{x}=-D^{-1} B \bar{x} .
$$

Hence

$$
\bar{x}=\left[\operatorname{diag}\left(\mathbf{1}-D^{-1} B \bar{x}\right)\right]^{-1}\left(-D^{-1} B\right) \bar{x} .
$$

We are reduced to find a fixed point for the application $H:[0,1]^{n}$ in $[0,1]^{n}$

$$
H(x)=\left[\operatorname{diag}\left(1-D^{-1} B x\right)\right]^{-1}\left(-D^{-1} B\right) x .
$$

Let be $A=D^{-1} B$ the next generation matrix. Since $\mathcal{R}_{0}=\rho\left(-D^{-1} B\right)$ and $A=-D^{-1} B$ is a nonnegative irreducible matrix, from the Perron-Frobenius Theorem there exists $v \gg 0$ such that

$$
A v=\mathcal{R}_{0} v .
$$

We choose $\varepsilon$ sufficiently small such that for any index $i$

$$
1+\varepsilon \mathcal{R}_{0} v_{i} \leq \mathcal{R}_{0}
$$


This is possible since $\mathcal{R}_{0}>1$. We deduce

$$
1 \leq \frac{\mathcal{R}_{0}}{1+\varepsilon \mathcal{R}_{0} v_{i}}
$$

and

$$
\varepsilon v_{i} \leq \frac{\mathcal{R}_{0} \varepsilon v_{i}}{1+\varepsilon \mathcal{R}_{0} v_{i}}=\frac{(A \varepsilon v)_{i}}{1+(A \varepsilon v)_{i}} .
$$

We have proved that there exists $\varepsilon>0$ such that $\varepsilon v \leq H(\varepsilon v)$. We also have $\varepsilon>1$ and $\varepsilon v \leq v$.

A similar argument shows that we can choose $\lambda$ with $0<\lambda v_{i} \leq 1$ and $\lambda$ large enough such that

$$
\frac{\mathcal{R}_{0}}{1+\mathcal{R}_{0} \lambda v_{i}} \leq 1
$$

which is equivalent to $\frac{\mathcal{R}_{0}-1}{\mathcal{R}_{0}} \leq \lambda v_{i}$. This implies $H(\lambda v) \leq \lambda v$. Choosing $\varepsilon \leq \lambda$ we have $\varepsilon v \leq H(\varepsilon v)$ and $H(\lambda v) \leq \lambda v \leq 1$. Since $H$ is a monotone function, $H$ maps the parallelepiped

$$
K=\{x \mid \varepsilon v \leq x \leq \lambda v\} \subset] 0,1\left[^{n},\right.
$$

into itself. By Brouwer fixed point Theorem we know that $H$ has a fixed point $\omega$ in $K$. This is an endemic equilibrium since $0 \ll \varepsilon v \leq \omega$.

It remains to show the uniqueness.

Lemma 2..1. If $\omega \gg 0$ is a strongly endemic equilibrium and if $\bar{x}$ is another equilibrium then $\bar{x} \leq \omega$.

Proof Let $\xi=\max _{i=1, \cdots, n} \frac{\bar{x}_{1}}{\omega_{1}}$. We have $\bar{x} \leq \xi \omega$ and there exists an index $i_{0}$ such that $\bar{x}_{i_{0}}=\xi \omega_{i_{0}}$. Since $A$ is nonnegative and $\bar{x}$ a fixed point of $H$ we have the following inequalities

$$
\bar{x}_{i_{0}}=\frac{(A \bar{x})_{i_{0}}}{1+(A \bar{x})_{i_{0}}} \leq \frac{(A \xi \omega)_{i_{0}}}{1+(A \xi \omega)_{i_{0}}}=\frac{\xi(A \omega)_{i_{0}}}{1+\xi(A \omega)_{i_{0}}} .
$$

By contradiction assume that $\xi>1$. From the last inequality we have

$$
\bar{x}_{i_{0}}<\frac{\xi(A \omega)_{i_{0}}}{1+(A \omega)_{i_{0}}} .
$$

But since $\bar{\omega}$ is a fixed point

$$
\bar{x}_{i_{0}}<\frac{\xi(A \omega)_{i_{0}}}{1+(A \omega)_{i_{0}}}=\xi \omega_{i_{0}}=\bar{x}_{i_{0}} .
$$

Therefore we obtain a contradiction.

We need a second lemma to end the proof. 
Lemma 2..2. If $\omega>0$ is an endemic equilibrium and if $A$ is irreducible then $\omega \gg 0$.

Proof Since $\omega>0$ and $A$ is irreducible, then we have $A \omega \gg 0$. Since components of $\omega$ are given by

$$
\omega_{j}=\frac{(A \omega)_{j}}{1+(A \omega)_{j}}>0,
$$

the assertion of the lemma is obtained.

The two lemmas prove that there exists an unique strongly endemic equilibrium.

\subsection{A theorem on stability}

To study the stability we need the following result which can be considered as a dual result to LaSalle's theorem [40].

Theorem 2..2. Let $G$ be an open set, containing the origin, which is positively invariant for the system $\dot{x}=A(x) . x$, where $A(x)$ is a Metzler matrix, depending continuously on $x$. We assume that there exists $c^{T} \gg 0$ such that $c^{T} A(x) \ll 0$ for any $x \in G, x \neq 0$. Then the origin is globally asymptotically stable on $G$.

Proof Let us consider on $G$ the Lyapunov function

$$
V(x)=\sum_{i=1}^{n} c_{i}\left|x_{i}\right| .
$$

We define $\varepsilon_{z}=\operatorname{sign}(z)$, i.e. $\left|x_{i}\right|=\varepsilon_{x_{i}} x_{i}$. This function is locally Lipschitz. The Dini derivative can be defined [40]. We have

$$
\begin{aligned}
\dot{V} & =\sum_{i=1}^{n} c_{i} \varepsilon_{x_{i}} \dot{x}_{i} \\
& =\sum_{i=1}^{n} c_{i} \varepsilon_{x_{i}} \sum_{j=1}^{n} a_{i j} x_{j} \\
& =\sum_{i=1}^{n} \sum_{j=1}^{n} c_{i} \varepsilon_{x_{i}} a_{i j} x_{j} \\
& =\sum_{j=1}^{n} \varepsilon_{x_{j}} x_{j} \sum_{i=1}^{n} c_{i} \varepsilon_{x_{j}} \varepsilon_{x_{i}} a_{i j} \\
& =\sum_{j=1}^{n} \varepsilon_{x_{j}} x_{j}\left[c_{j} a_{j j}+\sum_{i \neq j} c_{i} \varepsilon_{x_{j}} \varepsilon_{x_{i}} a_{i j}\right] \\
& \leq \sum_{j=1}^{n} \varepsilon_{x_{j}} x_{j}\left[c_{j} a_{j j}+\sum_{i \neq j} c_{i} a_{i j}\right]=\sum_{j=1}^{n}\left|x_{j}\right|\left(c^{T} A\right)_{j} \leq 0 .
\end{aligned}
$$


Since $c^{T} A(x) \ll 0$ on $G$, then the function $\dot{V}$ is negative definite. This ends the proof by the Lyapunov theorem.

\subsection{Global stability of the DFE}

We have the following result.

Theorem 2..3. The DFE of the system (2.1), which is the origin, is globally asymptotically stable if and only if $\mathcal{R}_{0} \leq 1$.

Proof Assume that $\mathcal{R}_{0}=\rho\left(-D^{-1} B\right) \leq 1$. We have seen from Proposition $2 . .1$ that this is equivalent to the stability of the matrix $D+N$. From the Perron-Frobenius theorem, since $D+B$ is irreducible, it follows that there exists an eigenvector $c \gg 0$ such that $(D+B)^{T} c=\alpha(D+B) c$. We choose the Lyapunov function

$$
V(x)=\langle c \mid x\rangle,
$$

positive definite on $\mathbb{R}_{+}^{n}$ and we have

$$
\dot{V}(x)=\left\langle(D+B)^{T} c \mid x\right\rangle-\langle\operatorname{diag}(x) B x \mid c\rangle \leq 0 .
$$

If $D+B$ is stable, i.e. $\alpha(D+B)<0$, the proof is finished, since this quantity is negative definite. It remains to study the case where $\alpha(D+B)=0$, or equivalently $\mathcal{R}_{0}=1$.

We apply Lasalle's invariance principle. We consider the largest invariant set contained in

$$
E=\{x \mid \operatorname{diag}(x) B x=0\} .
$$

The irreducibility of $B$ implies $\mathcal{L}=\{0\}$. Indeed if $x \in \mathcal{L} \subset E$ we have for all $(i, j)$,

$$
x_{i} \sum_{j} \beta_{i j} x_{j}=0 .
$$

The quantities are positive, this implies that for any couple of indices $\beta_{i j} x_{i} x_{j}=0$. By contradiction assume that $i_{0}$ is such that $x_{i_{0}} \neq 0$. There exists an index $i_{1}$ such that $\beta_{i_{1}, i_{0}} \neq 0$, from the irreducibility of $B$. It follows $x_{i_{1}}=0$. The trajectory $x(t)$ from $x$, satisfies for a small positive time $x(t)_{i_{0}} \neq 0$. Hence $x(t)_{i_{1}}=0$. By invariance of $\mathcal{L}$ we must have

$$
\dot{x}_{i_{1}}=-\sum_{j} \beta_{i_{1}, j} x_{j}=0 .
$$

Which in turn implies $x_{i_{2}}=0$ for any $\beta_{i_{1}, i_{2}} \neq 0$. In the other words, if the node $i_{2}$ is connected by an oriented path to the node $i_{1}$, then $x_{i_{2}}=0$. By a finite induction we deduce that we have $x_{i}=0$ for any node connected to the node $i_{1}$. Since by irreducibility [6] the graph associated to $B$ is strongly connected, we have $x_{i_{0}}=0$. This gives a contradiction. 


\subsection{Global stability of the endemic equilibrium}

Theorem 2..4. The endemic equilibrium of the system (2.1) is globally asymptotically stable on $\mathbb{R}^{n} \backslash\{0\}$ if and only if $\mathcal{R}_{0}>1$.

Proof Since $\mathcal{R}_{0}>1$, then there exists an unique equilibrium $\omega \gg 0$. We write (2.4) under new coordinates $x+X+\omega$. Using the definition of $\omega:(D+B) \omega-\operatorname{diag}(x) B x=0$, we get

$$
\dot{X}=[D+\operatorname{diag}(1-X-\omega) B-\operatorname{diag}(B \omega)] X .
$$

Since $\omega$ is in $] 0,1{ }^{n}$ which is an absorbing set, it is sufficient to consider (2.1) on this set, or equivalently, when $x \leq 1$. In this case $\operatorname{diag}(1-X-\omega)=\operatorname{diag}(1-x)$ and the matrix

$$
A(X)=D+\operatorname{diag}(1-X-\omega) B-\operatorname{diag}(B \omega)
$$

is Metzler. $X$ is in the compact set $-\omega+[0,1]^{n}$.

We apply (2..2). We know that for any irreducible $B \geq 0$, for any Metzler stable $D$ such that $\rho\left(-D^{-1} B\right)>1$ there exists $\omega \gg 0$ such that

$$
(D-\operatorname{diag}(B \omega)+B) \omega=0 .
$$

In other words $A(-\omega)$ satisfies $A(-\omega) \omega=0$. From Proposition (2..1) we deduce $\alpha(A(-\omega))=0$. Since this matrix is irreducible, and transposing, we know that there exists $c \gg 0$ such that

$$
c^{T} A(-\omega)=c^{T}(D-\operatorname{diag}(B \omega)+B)=0 .
$$

Then for $X+\omega \gg 0$ (i.e. $x \gg 0$, we have

$$
c^{T} A(X)=-c^{T}(X+\omega) B \ll 0 .
$$

This proves the stability on $] 0,1\left[^{n}\right.$. Since the vector field is strictly entrant, this ends the proof on $\mathbb{R}^{n} \backslash\{0\}$.

\section{A differential susceptibility and infectivity model}

We consider the following model

$$
\left\{\begin{array}{l}
\dot{S}=\Lambda-\mu S-\operatorname{diag}(B I) S \\
\dot{I}=\langle B I \mid S\rangle e_{1}+A I,
\end{array}\right.
$$

where $S \in \mathbb{R}_{+}^{n}$ is the state of susceptible individuals and $I \in \mathbb{R}_{+}^{k}$ is the state of infectious. The matrix $B \geq 0$ represents the coefficients of infectivity, actually $B(i, j)$ is the contact and infectivity of $I_{j}$ in the group $S_{i}$. As usual, $e_{1}$ is the first vector of the canonical basis of $\mathbb{R}^{k}$. Finally, $A$ is a stable Metzler matrix and represents the evolution through the infectious stages. This model 
encompass known models of DI, SP, or differential susceptibility models . We generalize the results obtained in $[28,24]$.

It is straightforward to check that the nonnegative orthant is positively invariant by this system, that there exists a compact positively invariant absorbing set. The DFE is given by $\left(S^{*}, 0\right) \in$ $\mathbb{R}_{+}^{n} \times \mathbb{R}_{+}^{k}$ where $S^{*}=\frac{1}{\mu} \Lambda$.

\subsection{Basic reproduction ratio}

We can give a simple elegant formula for the $\mathcal{R}_{0}$ (compare with [28, 24]). To obtain $\mathcal{R}_{0}$ we can use the techniques developed in [67]. We claim that

$$
\mathcal{R}_{0}=\left\langle B\left(-A^{-1}\right) e_{1} \mid S^{*}\right\rangle .
$$

We use the expression $\left(-A^{-1}\right)$ to put the emphasis on the fact that $\left(-A^{-1}\right)>0$ because $A$ is Metzler stable. Using the framework of [67], we denote by $\mathcal{F}_{i}(S, I)$ the rate of appearance of new infections in compartment $i$, and by $\mathcal{V}_{i}(S, I)$ the rate of transfer of individuals in and out the compartment $i$ by all other means. The matrix $\mathcal{V}$ is the "mass" balance of the compartments. Note that our $\mathcal{V}$ is the opposite of the same used in [67]. Then

$$
\mathcal{F}(S, I)=\left[\begin{array}{c}
0 \\
\langle B I \mid S\rangle e_{1}
\end{array}\right]
$$

and

$$
\mathcal{V}(S, I)=\left[\begin{array}{c}
\Lambda-\mu S-\operatorname{diag}(B I) S \\
A I
\end{array}\right]
$$

The Jacobian matrices are

$$
D \mathcal{F}(x, y)=\left[\begin{array}{cc}
0 & 0 \\
e_{1}(B I)^{T} & e_{1} S^{T} B
\end{array}\right], \quad D \mathcal{V}(x, y)=\left[\begin{array}{cc}
-\mu I-\operatorname{diag}(B I) & -\operatorname{diag}(S) B \\
0 & A
\end{array}\right]
$$

Noting that we have sorted the variables in the reverse order in comparison with [67], we set $F=x^{*} \mathbf{b} \beta^{T}$ and $V=A$. It is proved in [67] that the basic reproduction number is the spectral radius of the next generation matrix for the model, namely $-F V^{-1}$ computed at the DFE (the minus sign comes from Metzler matrices used in place of $M$-matrices),

$$
\mathcal{R}_{0}=\rho\left(-F V^{-1}\right)=\rho\left(e_{1} S^{* T} B\left(-A^{-1}\right)\right) .
$$

It is clear that $e_{1} S^{* T} B\left(-A^{-1}\right)$ is a rank one matrix, the only nonzero eigenvalue is given by $S^{* T} B\left(-A^{-1}\right) e_{1}$, which is exactly our claim.

\subsection{Global stability of the DFE}

We have the following theorem. 
Theorem 3..1. If $\mathcal{R}_{0} \leq 1$ then the DFE is globally asymptotically stable on the nonnegative orthant. If $\mathcal{R}_{0}>1$ the DFE is unstable.

Proof We will introduce some notation to simplify the exposition of the proof. Actually these notations are used in MATLAB and SCILAB. For two matrices $M$ and $N$ of same size we denote by $D=M . / N$ the matrix which is defined by $D(i, j)=M(i, j) / N(i, j)$. In the same spirit $L=\ln M$ will denote the matrix defined by $L(i, j)=\ln (M(i, j))$. We can now define the Lyapunov-LaSalle function on $\mathbb{R}_{+}^{n} \times \mathbb{R}_{+}^{k} \backslash\left\{S^{*}\right\} \times \mathbb{R}_{+}^{k}$ :

$$
V_{D F E}(S, I)=\mathcal{R}_{0}\left\langle\mathbf{1} \mid S-S^{*}\right\rangle-\mathcal{R}_{0}\left\langle S^{*} \mid \ln S-\ln S^{*}\right\rangle+\left\langle B\left(-A^{-1}\right) I \mid S^{*}\right\rangle .
$$

We have, using the fact that $\Lambda=\mu S^{*}$ :

$$
\begin{aligned}
\dot{V}_{D F E}=\mu \mathcal{R}_{0}\left\langle\mathbf{1} \mid S^{*}\right\rangle-\mathcal{R}_{0}\langle\mathbf{1} \mid \operatorname{diag}(B I) S\rangle-\mu \mathcal{R}_{0}\langle\mathbf{1} \mid S\rangle \\
-\mu \mathcal{R}_{0}\left\langle S^{*} . / S \mid S^{*}\right\rangle+\mathcal{R}_{0}\left\langle\operatorname{diag}(B I) \mathbf{1} \mid S^{*}\right\rangle+\mu \mathcal{R}_{0}\left\langle\mathbf{1} \mid S^{*}\right\rangle \\
+\langle B I \mid S\rangle\left\langle B\left(-A^{-1}\right) e_{1} \mid S^{*}\right\rangle-\left\langle B I \mid S^{*}\right\rangle .
\end{aligned}
$$

Taking into account the formula (3.2) on $\mathcal{R}_{0}$ with the relations

$$
\langle\mathbf{1} \mid \operatorname{diag}(B I) S\rangle=\langle B I \mid S\rangle,\left\langle\operatorname{diag}(B I) \mathbf{1} \mid S^{*}\right\rangle=\left\langle B I \mid S^{*}\right\rangle
$$

and $\langle\mathbf{1} \mid S\rangle=\left\langle S^{*} \mid S . / S^{*}\right\rangle$ the preceding equation becomes

$$
\dot{V}_{D F E}=\mu \mathcal{R}_{0}\left\langle\mathbf{2}-S^{*} . / S-S . / S^{*} \mid S^{*}\right\rangle+\left(\mathcal{R}_{0}-1\right)\left\langle B I \mid S^{*}\right\rangle .
$$

The inequality between the arithmetic and the geometric means and $\mathcal{R}_{0} \leq 1$ imply $\dot{V}_{D F E} \leq 0$. The largest invariant set contained in the set $\left\{(S, I) \mid \dot{V}_{D F E}(S, I)=0\right\}$ satisfies the relation $S=S^{*}$. Since $A$ is a stable Metzler matrix, by Lasalle's invariance principle [40] the DFE is globally asymptotically stable. This ends the proof

\subsection{Endemic equilibrium}

Theorem 3..2. There exists a unique endemic equilibrium in the nonnegative orthant if and only if $\mathcal{R}_{0}>1$.

Proof. We look for an equilibrium $(\bar{S}, \bar{I})$ with $\bar{I}>0$. From the relations

$$
\left\{\begin{array}{l}
0=\Lambda-\mu \bar{S}-\operatorname{diag}(\bar{B} \bar{I}) \bar{S}, \\
0=\langle\bar{B} \bar{I} \mid \bar{S}\rangle e_{1}+A \bar{I}
\end{array}\right.
$$

we deduce, since $A$ is Metzler stable, that $\bar{I}=\langle\bar{B} \bar{I} \mid \bar{S}\rangle\left(-A^{-1}\right) e_{1}$. From the second relation of (3.4) and taking the inner product with $e_{1}$ we obtain $\langle\bar{B} \bar{I} \mid \bar{S}\rangle=-\left\langle A \bar{I} \mid e_{1}\right\rangle$. Finally 


$$
\bar{I}=-\left\langle A \bar{I} \mid e_{1}\right\rangle\left(-A^{-1}\right) e_{1} .
$$

Then to compute $\bar{I}$ it is sufficient to find $-\left\langle A \bar{I} \mid e_{1}\right\rangle$.

Again with the expression $\bar{I}=\langle\bar{B} \bar{I} \mid \bar{S}\rangle\left(-A^{-1}\right) e_{1}$, we get

$$
\langle\bar{B} \bar{I} \mid \bar{S}\rangle=\langle\bar{B} \bar{I} \mid \bar{S}\rangle\left\langle B\left(-A^{-1}\right) e_{1} \mid \bar{S}\right\rangle .
$$

If $\langle\bar{B} \bar{I} \mid \bar{S}\rangle \neq 0$ then we have

$$
\left\langle B\left(-A^{-1}\right) e_{1} \mid \bar{S}\right\rangle=1
$$

From the first equation in (3.4) we have

$$
\bar{S}=[\operatorname{diag}(\mu \mathbf{1}+B \bar{I})]^{-1} \Lambda=\left[\operatorname{diag}\left(\mathbf{1}+\frac{1}{\mu} B \bar{I}\right)\right]^{-1} S^{*} .
$$

Using this value of $\bar{S}$ and of $B \bar{I}$ in (3.6) gives

$$
\left\langle B\left(-A^{-1}\right) e_{1} \mid\left[\operatorname{diag}\left(\mathbf{1}-\frac{\left\langle A \bar{I} \mid e_{1}\right\rangle}{\mu} B\left(-A^{-1}\right) e_{1}\right)\right]^{-1} S^{*}\right\rangle=1 .
$$

In other words $-\left\langle A \bar{I} \mid e_{1}\right\rangle$ is a solution of $H(x)=1$ with

$$
H(x)=\left\langle B\left(-A^{-1}\right) e_{1} \mid\left[\operatorname{diag}\left(\mathbf{1}+\frac{x}{\mu} B\left(-A^{-1}\right) e_{1}\right)\right]^{-1} S^{*}\right\rangle .
$$

It is clear that $H(x)$ is a strictly decreasing function satisfying $\lim _{x \rightarrow+\infty} H(x)=0$. Then a unique positive solution exists if and only if $H(0)>1$. Since $H(0)=\mathcal{R}_{0}$ we have a positive solution. Since, from (3.7) we have $\bar{S} \gg 0$ and from (3.5), with $-\left\langle A \bar{I} \mid e_{1}\right\rangle>0, \bar{I}>0$, then the equilibrium is endemic. Moreover $\langle\bar{B} \bar{I} \mid \bar{S}\rangle \neq 0>0$. From the preceding analysis we see that if $\mathcal{R}_{0}=1$ then the unique equilibrium is the DFE. In the case $\mathcal{R}_{0}<1$ we have $\bar{I}<0$, that is the equilibrium is not biologically feasible.

\subsection{Global stability of the endemic equilibrium}

To prove the global stability of the endemic equilibrium we need to study in more detail the structure of $A$. We will treat in this section one example. For the sake of brevity we will consider a model of two susceptible classes and two infective classes with stage progression. It is not difficult, but certainly more involved, to treat exactly in the same way the case of $n$ susceptible compartments and $k$ infectious compartments. The compartimental model is represented in figure 3.4..

The model is given by the following system of ordinary differential equations. 


$$
\left\{\begin{array}{l}
\dot{S}_{1}=p_{1} \Lambda-\mu S_{1}-\beta_{11} I_{1} S_{1}-\beta_{12} I_{2} S_{1} \\
\dot{S}_{2}=p_{2} \Lambda-\mu S_{2}-\beta_{21} I_{1} S_{2}-\beta_{22} I_{2} S_{2} \\
\dot{I}_{1}=\beta_{11} I_{1} S_{1}+\beta_{12} I_{2} S_{1}+\beta_{21} I_{1} S_{2}+\beta_{22} I_{2} S_{2}-\left(\mu+\gamma_{1}+\delta_{1}\right) I_{1} \\
\dot{I}_{2}=\gamma_{1} I_{1}-\left(\mu+\gamma_{1}+\delta_{2}\right) I_{2} \\
\dot{R}=\gamma_{2} I_{2}-\mu R .
\end{array}\right.
$$

Theorem 3..3. If $\mathcal{R}_{0}>1$, then the unique endemic equilibrium is globally asymptotically stable. The basic reproduction number is given by

$$
\mathcal{R}_{0}=\frac{\gamma_{1}\left(\beta_{12} S_{1}^{*}+\beta_{22} S_{2}^{*}\right)+\left(\mu+\gamma_{1}+\delta_{2}\right)\left(\beta_{11} S_{1}^{*}+\beta_{21} S_{2}^{*}\right)}{\left(\mu+\gamma_{1}+\delta_{1}\right)\left(\mu+\gamma_{1}+\delta_{2}\right)}
$$

Proof. The basic reproduction ratio is obtained by applying (3.2). From the general theory we know that there exists a unique endemic equilibrium which satisfies the following relations

$$
\left\{\begin{array}{l}
p_{1} \Lambda=\mu \bar{S}_{1}+\beta_{11} \bar{I}_{1} \bar{S}_{1}+\beta_{12} \bar{I}_{2} \bar{S}_{1} \\
p_{2} \Lambda=\mu \bar{S}_{2}+\beta_{21} \bar{I}_{1} \bar{S}_{2}+\beta_{22} \bar{I}_{2} \bar{S}_{2} \\
\beta_{11} \bar{I}_{1} \bar{S}_{1}+\beta_{12} \bar{I}_{2} \bar{S}_{1}+\beta_{21} \bar{I}_{1} \bar{S}_{2}+\beta_{22} \bar{I}_{2} \bar{S}_{2}=\left(\mu+\gamma_{1}+\delta_{1}\right) \bar{I}_{1} \\
\gamma_{1} \bar{I}_{1}=\left(\mu+\gamma_{1}+\delta_{2}\right) \bar{I}_{2} \\
\gamma_{2} \bar{I}_{2}=\mu \bar{R}
\end{array}\right.
$$

Let us consider a possible Lyapunov function

$$
V_{E E}=\left(S_{1}-\bar{S}_{1} \ln S_{1}\right)+\left(S_{2}-\bar{S}_{2} \ln S_{2}\right)+\left(I_{1}-\bar{I}_{1} \ln I_{1}\right)+\left(\frac{\beta_{12} \bar{S}_{1}+\beta_{22} \bar{S}_{2}}{\left(\mu+\gamma_{1}+\delta_{2}\right)}\right)\left(I_{2}-\bar{I}_{2} \ln I_{2}\right)
$$

Setting $d=\frac{\beta_{12} \bar{S}_{1}+\beta_{22} \bar{S}_{2}}{\left(\mu+\gamma_{1}+\delta_{2}\right)}$, its derivative along the trajectories of (3.8) is

$$
\begin{aligned}
\dot{V}_{E E} & =\left[p_{1} \Lambda-\mu S_{1}-\beta_{11} I_{1} S_{1}-\beta_{12} I_{2} S_{1}-p_{1} \Lambda \frac{\bar{S}_{1}}{S_{1}}+\mu \bar{S}_{1}+\beta_{11} I_{1} \bar{S}_{1}+\beta_{12} I_{2} \bar{S}_{1}\right] \\
& +\left[p_{2} \Lambda-\mu S_{2}-\beta_{21} I_{1} S_{2}-\beta_{22} I_{2} S_{2}-p_{2} \Lambda \frac{\bar{S}_{2}}{S_{2}}+\mu \bar{S}_{2}+\beta_{21} I_{1} \bar{S}_{2}+\beta_{22} I_{2} \bar{S}_{2}\right] \\
& +\left[\beta_{11} I_{1} S_{1}+\beta_{12} I_{2} S_{1}+\beta_{21} I_{1} S_{2}+\beta_{22} I_{2} S_{2}-\left(\mu+\gamma_{1}+\delta_{1}\right) I_{1}\right. \\
& \left.-\beta_{11} \bar{I}_{1} S_{1}-\beta_{12} I_{2} \frac{\bar{I}_{1}}{I_{1}} S_{1}-\beta_{21} \bar{I}_{1} S_{2}-\beta_{22} I_{2} \frac{\bar{I}_{1}}{I_{1}} S_{2}+\left(\mu+\gamma_{1}+\delta_{1}\right) \bar{I}_{1}\right] \\
& +d\left[\gamma_{1} I_{1}-\left(\mu+\gamma_{1}+\delta_{2}\right) I_{2}-\gamma_{1} I_{1} \frac{\bar{I}_{2}}{I_{2}}+\left(\mu+\gamma_{1}+\delta_{2}\right) \bar{I}_{2}\right] .
\end{aligned}
$$

By using the endemic relations in the system (3.9) we obtain, 


$$
\begin{aligned}
\dot{V}_{E E} & =\left[\mu \bar{S}_{1}+\beta_{11} \bar{I}_{1} \bar{S}_{1}+\beta_{12} \bar{I}_{2} \bar{S}_{1}-\mu \bar{S}_{1} \frac{S_{1}}{\bar{S}_{1}}\right. \\
& \left.-\left(\mu \bar{S}_{1}+\beta_{11} \bar{I}_{1} \bar{S}_{1}+\beta_{12} \bar{I}_{2} \bar{S}_{1}\right) \frac{\bar{S}_{1}}{S_{1}}+\mu \bar{S}_{1}+\beta_{11} I_{1} \bar{S}_{1}+\beta_{12} I_{2} \bar{S}_{1}\right] \\
& +\left[\mu \bar{S}_{2}+\beta_{21} \bar{I}_{1} \bar{S}_{2}+\beta_{22} \bar{I}_{2} \bar{S}_{2}-\mu \bar{S}_{2} \frac{S_{2}}{S_{2}}\right. \\
& \left.-\left(\mu \bar{S}_{2}+\beta_{21} \bar{I}_{1} \bar{S}_{2}+\beta_{22} \bar{I}_{2} \bar{S}_{2}\right) \frac{\bar{S}_{2}}{S_{2}}+\mu \bar{S}_{2}+\beta_{21} I_{1} \bar{S}_{2}+\beta_{22} I_{2} \bar{S}_{2}\right]-\left(\mu+\gamma_{1}+\delta_{1}\right) I_{1} \\
& -\beta_{11} \bar{I}_{1} \bar{S}_{1} \frac{S_{1}}{\bar{S}_{1}}-\beta_{12} \bar{I}_{2} \bar{S}_{1} \frac{S_{1}}{\bar{S}_{1}} \frac{\bar{I}_{1}}{I_{1}} \bar{I}_{2}+\beta_{21} \bar{I}_{1} \bar{S}_{2} \frac{S_{2}}{\bar{S}_{2}}-\beta_{22} \bar{I}_{2} \bar{S}_{2} \frac{S_{2}}{\bar{S}_{2}} \frac{\bar{I}_{1}}{I_{1}} \frac{I_{2}}{\bar{I}_{2}} \\
& +\beta_{11} \bar{I}_{1} \bar{S}_{1}+\beta_{12} \bar{I}_{2} \bar{S}_{1}+\beta_{21} \bar{I}_{1} \bar{S}_{2}+\beta_{22} \bar{I}_{2} \bar{S}_{2} \\
& +d\left[\gamma_{1} I_{1}-\left(\mu+\gamma_{1}+\delta_{2}\right) I_{2}-\gamma_{1} I_{1} \frac{\bar{I}_{2}}{I_{2}}+\gamma_{1} \bar{I}_{1}\right] \\
& =\mu \bar{S}_{1}\left[2-\frac{\bar{S}_{1}}{S_{1}}-\frac{S_{1}}{\bar{S}_{1}}\right]+\mu \bar{S}_{2}\left[2-\frac{\bar{S}_{2}}{S_{2}}-\frac{S_{2}}{\bar{S}_{2}}\right] \\
& \left.+\beta_{11} \bar{I}_{1} \bar{S}_{1}\left[2-\frac{\bar{S}_{1}}{S_{1}}-\frac{S_{1}}{S_{1}}\right]+\beta_{12} \bar{I}_{2} \bar{S}_{1}\right)\left[2-\frac{\bar{S}_{1}}{S_{1}}-\frac{S_{1}}{S_{1}} \frac{\bar{I}_{1}}{I_{1}} \frac{I_{2}}{I_{2}}\right] \\
& +\beta_{21} \bar{I}_{1} \bar{S}_{2}\left[2-\frac{\bar{S}_{2}}{S_{2}}-\frac{S_{2}}{S_{2}}\right]+\beta_{22} \bar{I}_{2} \bar{S}_{2}\left[2-\frac{\bar{S}_{2}}{S_{2}}-\frac{S_{2}}{S_{2}} \frac{\bar{I}_{1}}{I_{1}} \frac{I_{2}}{I_{2}}\right] \\
& +\left(\beta_{11} \bar{S}_{1}+\beta_{21} \bar{S}_{2}+d \gamma_{1}-\left(\mu+\gamma_{1}+\delta_{1}\right)\right) I_{1} \\
& +\left(\beta_{12} \bar{S}_{1}+\beta_{22} \bar{S}_{2}-d\left(\mu+\gamma_{1}+\delta_{2}\right)\right) I_{2}-d \gamma_{1} \frac{\bar{I}_{1}}{I_{1}} \frac{I_{2}}{I_{2}}+d \gamma_{1} \bar{I}_{1} .
\end{aligned}
$$

Using the expression for $d$, we observe that

$$
\beta_{12} \bar{S}_{1}+\beta_{22} \bar{S}_{2}-d\left(\mu+\gamma_{1}+\delta_{2}\right)=0
$$

and

$$
\begin{aligned}
\beta_{11} \bar{S}_{1}+\beta_{21} \bar{S}_{2}+d \gamma_{1}-\left(\mu+\gamma_{1}+\delta_{1}\right) & =\beta_{11} \bar{S}_{1}+\beta_{21} \bar{S}_{2}+\frac{\beta_{12} \bar{S}_{1}+\beta_{22} \bar{S}_{2}}{\left(\mu+\gamma_{1}+\delta_{2}\right)} \gamma_{1}-\left(\mu+\gamma_{1}+\delta_{1}\right) \\
& =\frac{\left(\mu+\gamma_{1}+\delta_{2}\right)\left(\beta_{11} \bar{S}_{1}+\beta_{21} \bar{S}_{2}\right)+\gamma_{1}\left(\beta_{12} \bar{S}_{1}+\beta_{22} \bar{S}_{2}\right)}{\left(\mu+\gamma_{1}+\delta_{2}\right)}-\left(\mu+\gamma_{1}+\delta_{1}\right) \\
& =\left(\mu+\gamma_{1}+\delta_{1}\right)\left[\frac{\left(\mu+\gamma_{1}+\delta_{2}\right)\left(\beta_{11} \bar{S}_{1}+\beta_{21} \bar{S}_{2}\right)+\gamma_{1}\left(\beta_{12} \bar{S}_{1}+\beta_{22} \bar{S}_{2}\right)}{\left(\mu+\gamma_{1}+\delta_{1}\right)\left(\mu+\gamma_{1}+\delta_{2}\right)}-1\right] \\
& =0 .
\end{aligned}
$$

Substituting the endemic relations in the third equation of system (3.9), we obtain

$$
\begin{gathered}
\beta_{11} \bar{I}_{1} \bar{S}_{1}+\beta_{12} \bar{I}_{2} \bar{S}_{1}+\beta_{21} \bar{I}_{1} \bar{S}_{2}+\beta_{22} \bar{I}_{2} \bar{S}_{2}=\left(\mu+\gamma_{1}+\delta_{1}\right) \bar{I}_{1} \\
\left(\beta_{11} \bar{S}_{1}+\beta_{21} \bar{S}_{2}+\frac{\gamma_{1}}{\left(\mu+\gamma_{1}+\delta_{2}\right)}\left(\beta_{12} \bar{S}_{1}+\beta_{22} \bar{S}_{2}\right)\right)=\left(\mu+\gamma_{1}+\delta_{1}\right) \\
\frac{\left(\mu+\gamma_{1}+\delta_{2}\right)\left(\beta_{11} \bar{S}_{1}+\beta_{21} \bar{S}_{2}\right)+\gamma_{1}\left(\beta_{12} \bar{S}_{1}+\beta_{22} \bar{S}_{2}\right)}{\left(\mu+\gamma_{1}+\delta_{1}\right)\left(\mu+\gamma_{1}+\delta_{2}\right)}=1 \\
d \gamma_{1} \bar{I}_{1}=\frac{\beta_{12} \bar{S}_{1}+\beta_{22} \bar{S}_{2}}{\left(\mu+\gamma_{1}+\delta_{2}\right)} \gamma_{1} \bar{I}_{1} \\
=\frac{\gamma_{1}}{\left(\mu+\gamma_{1}+\delta_{2}\right)}\left(\beta_{12} \bar{I}_{1} \bar{S}_{1}+\beta_{22} \bar{I}_{1} \bar{S}_{2}\right) \\
=\beta_{12} \bar{I}_{2} \bar{S}_{1}+\beta_{22} \bar{I}_{2} \bar{S}_{2} .
\end{gathered}
$$


and finally we have the following expression for $\dot{V}_{E E}$

$$
\begin{aligned}
\dot{V}_{E E} & =\mu \bar{S}_{1}\left[2-\frac{\bar{S}_{1}}{S_{1}}-\frac{S_{1}}{\bar{S}_{1}}\right]+\mu \bar{S}_{2}\left[2-\frac{\bar{S}_{2}}{S_{2}}-\frac{S_{2}}{\bar{S}_{2}}\right] \\
& \left.+\beta_{11} \bar{I}_{1} \bar{S}_{1}\left[2-\frac{\bar{S}_{1}}{S_{1}}-\frac{S_{1}}{S_{1}}\right]+\beta_{12} \bar{I}_{2} \bar{S}_{1}\right)\left[3-\frac{\bar{S}_{1}}{S_{1}}-\frac{S_{1}}{S_{1}} \frac{\bar{I}_{1}}{I_{1}} \frac{I_{2}}{I_{2}}-\frac{I_{1}}{I_{1}} \frac{\bar{I}_{2}}{I_{2}}\right] \\
& +\beta_{21} \bar{I}_{1} \bar{S}_{2}\left[2-\frac{\bar{S}_{2}}{S_{2}}-\frac{S_{2}}{\bar{S}_{2}}\right]+\beta_{22} \bar{I}_{2} \bar{S}_{2}\left[3-\frac{\bar{S}_{2}}{S_{2}}-\frac{S_{2}}{\bar{S}_{2}} \bar{I}_{1} \frac{I_{2}}{I_{1}}-\frac{I_{1}}{I_{1}} \bar{I}_{2}\right. \\
& \leq 0
\end{aligned}
$$

Using the comparison between the arithmetical and the geometrical means we see that $\dot{V}_{E E}$ is negative definite. This ends the proof of the theorem.

\section{References}

[1] P. Adda, J. Dimi, A. Iggidr, J. Kamgang, G. Sallet, J. Tewa.General models of host-parasite systems. Global Analysis, DCDS series B, 8 (2007), 1-17.

[2] J. Arino, C. C. McCluskey, P. van den Driessche.Global results for an epidemic model with vaccination that exhibits backward bifurcation. SIAM J. Appl. Math., 64 (2003), 260-276.

[3] N. Bame, S. Bowong, J. Mbang, J., G. Sallet, J. Tewa. Global stability analysis for SEIS models with $n$ latent classes. Math. Biosci. Eng., (to appear).

[4] E. Beretta, V. Capasso. On the general structure of epidemic systems. Global asymptotic stability. Comput. Math. Appl., Part A, 12 (1986), 677-694.

[5] E. Beretta, Y. Takeuchi. Global stability of Lotka-Volterra di? usion models with continuous time delay. SIAM J. Appl. Math., 48 (1988), 627-651.

[6] A. Berman, R. J. Plemmons. Nonnegative matrices in the mathematical sciences. SIAM, 1994.

[7] S. Busenberg, P. van den Driessche. A method for proving the nonexistence of limit cycles. J. Math. Anal. Appl., 172 (1993), 463-479.

[8] O. Diekmann, J. A. P. Heesterbeek. Mathematical epidemiology of infectious diseases, Wiley Series in Mathematical and Computational Biology, John Wiley \& Sons Ltd., Chichester, 2000. Model building, analysis and interpretation.

[9] O. Diekmann, J. A. P. Heesterbeek, J. A. J. Metz. On the definition and the computation of the basic reproduction ratio $R 0$ in models for infectious diseases in heterogeneous populations. J. Math. Biol., 28 (1990), 365-382.

[10] K. Dietz. The estimation of the basic reproduction number for infectious diseases. Statist. Meth. Med. Res., (1993), 23-41. 
[11] L. Esteva, C. Vargas. A model for dengue disease with variable human population. J. Math. Biol., (1998), 220-240.

[12] B. S. Goh. Global stability in manyspecies systems. Amer.Naturalist, (1977), 135-143.

[13] A. Gumel, C. C. McCluskey, P. van den Driessche. Mathematical study of a staged progression HIV model with imperfect vaccine. J. Theoret. Biol., 68 (2006), 2105-2128.

[14] A. Gumel, C. C. McCluskey, J. Watmough. An sveir model for assessing the potential impact of an imperfect anti-sars vaccine. Math. Biosci. Eng., 3 (2006).

[15] H. Guo, M. Li. Global dynamics of a staged progression model for infectious diseases. Math. Biosci. Eng., 3 (2006), 513-525.

[16] G. W. Harrison. Global stability of predator-prey interactions. J. Math. Biol., 8 (1979), 159171.

[17] J. A. P. Heesterbeek. A brief history of RO and a recipe for its calculation. Acta Biotheorica, 50 (2002), 189-204.

[18] J. A. P. Heesterbeek, K. Dietz. The concept of RO in epidemic theory. Statist. Neerlandica, 50 (1996), 89-110.

[19] H. W. Hethcote. Qualitative analyses of communicable disease models. Math. Biosci., 28 (1976), 335-356.

[20] H. W. Hethcote. The mathematics of infectious diseases. SIAM Rev., 42 (2000), 599-653 (electronic).

[21] H. W. Hethcote, H. R. Thieme. Stability of the endemic equilibrium in epidemic models with subpopulations. Math. Biosci., 75 (1985), 205- 227.

[22] M. Hirsch. Systems of differential equations that are competitive or cooperative. iv: Structural stability in three-dimensional systems. SIAM J. Appl. Math., 21 (1990), 1125-1234.

[23] J. Hyman, J. Li. An intuitive formulation for the reproductive number for the spread of diseases in heterogeneous populations. Math. Biosci., 167 (2000).

[24] J. Hyman, J. Li.Differential susceptibility and infectivity epidemic models. J. Math. Biol., 50 (2005),626-644.

[25] J. Hyman, J. Li, E. Stanley. The initialization and sensitivity of multi-group models for the transmission of HIV. J. Theoret. Biol., 208 (2001), 227-249.

[26] J. M. Hyman, J. Li. Threshold conditions for the spread of the HIV infection in age structured populations of homosexual men. J.theor. Biol., 166 (1994), 9-31. 
[27] J. M. Hyman,J. Li. The reproductive number for an HIV model with differential infectivity and staged progression. Linear Algebra Appl., 398 (2005), 101-116.

[28] J. M. Hyman, J. Li. Differential susceptibility and infectivity epidemic models. Math. Biosci. Eng., 3 (2006).

[29] J. M. Hyman, J. Li, E. Stanley. The differential infectivity and staged progression models for the transmission of HIV. Math. Biosci., 155 (1999), 77-109.

[30] A. Iggidr, J. Kamgang, G. Sallet, J. Tewa. Global analysis of new malaria intra-host models with a competitive exclusion principle. SIAM J. Appl. Math., 67 (2006), 260-278.

[31] A. Iggidr, J. Mbang, G. Sallet. Stability analysis of within-host parasite models with delays. Math. Biosci., available online (2007).

[32] A. Iggidr, J. Mbang, G. Sallet, J. Tewa. Multi-compartment models. DCDS series B, to appear (2007).

[33] J. A. Jacquez, C. P. Simon. Qualitative theory of compartmental systems. SIAM Rev., 35 (1993), 43-79.

[34] J. A. Jacquez, C. P. Simon, J. Koopman. The reproduction number in deterministic models of contagious diseases. Comment. Theor. Biol., 2 (1991).

[35] J. A. Jacquez, C. P. Simon, J. Koopman, L. Sattenspiel, T. Perry. Modeling and analyzing HIV transmission : the effect of contact patterns. Math. Biosci., 92 (1988).

[36] A. Korobeinikov. Lyapunov functions and global properties for SEIR and SEIS models. Math. Med. Biol., 21 (2004), 75-83.

[37] A. Korobeinikov, P. Maini. A Lyapunov function and global properties for SIR and SEIR epidemiolgical models with nonlinear incidence. Math. Biosci. Eng., 1 (2004), 57-60.

[38] A. Korobeinikov, P. Maini. Nonlinear incidence and stability of infectious diseases. Math. Med. Biol., 22 (2005), 113-128.

[39] A. Lajmanovich, J. Yorke. A deterministic model for gonorrhea in a nonhomogeneous population. Math. Biosci., 28 (1976), 221-236.

[40] J. P. LaSalle. The stability of dynamical systems. Society for Industrial and Applied Mathematics, Philadelphia, Pa., 1976. With an appendix: "Limiting equations and stability of nonautonomous ordinary differential equations" by Z. Artstein, Regional Conference Series in Applied Mathematics.

[41] M. Li, J. S. Muldowney. On r.a. Smith's autonomous convergence theorem. Rocky Mountain J. Math., 25 (1995), 365-379. 
[42] M. Y. Li, J. R. Graef, L. Wang, J. Karsai. Global dynamics of a SEIR model with varying total population size. Math. Biosci., 160 (1999), 191-213.

[43] M. Y. Li, J. S. Muldowney. Global stability for the SEIR model in epidemiology. Math. Biosci., 125 (1995), 155-164.

[44] M. Y. Li, J. S. Muldowney. A geometric approach to global stability problems. SIAM J. Math. Anal., 27 (1996), 1070-1083.

[45] M. Y. Li, J. S. Muldowney, P. van den Driessche. Global stability of SEIRS models in epidemiology. Canad. Appl. Math. Quart., 7 (1999), 409-425.

[46] M. Y. Li, H. L. Smith, L. Wang. Global dynamics an SEIR epidemic model with vertical transmission. SIAM J. Appl. Math., 62 (2001), 58-69 (electronic).

[47] M. Y. Li, L. Wang. Global stability in some SEIR epidemic models, in Mathematical approaches for emerging and reemerging infectious diseases: models, methods, and theory. (Minneapolis, MN, 1999), vol. 126 of IMA Vol. Math. Appl., Springer, New York, (2002), 295-311.

[48] Y. Li, J. S. Muldowney. On Bendixson's criterion. J. Differ. Equations, 106 (1993), 27-39.

[49] X. Lin, J. W.H. So. Global stability of the endemic equilibrium and uniform persistence in epidemic models with subpopulations. J. Aust. Math. Soc., Ser. B, 34 (1993), 282-295.

[50] W. M. Liu, H. W. Hethcote, S. A. Levin. Dynamical behavior of epidemiological models with nonlinear incidence rates. J. Math. Biol., 25 (1987), 359-380.

[51] D. G. Luenberger. Introduction to dynamic systems. Theory, models, and applications. John Wiley \& Sons Ltd., 1979.

[52] Z. Ma, J. Liu, J. Li. Stability analysis for differential infectivity epidemic models. Nonlinear Anal. : Real world applications, (2003), 841- 856.

[53] C. McCluskey. A model of HIV/AIDS with staged progression and amelioration. Math. Biosci., 181 (2003), 1-16.

[54] C. McCluskey, P. van den Driessche. Global analysis of two tuberculosis models. J. Dyn. Differ. Equations, 16 (2004), 139-166.

[55] C. C. McCluskey. Lyapunov functions for tuberculosis models with fast and slow progression. Math. Biosci. Eng., to appear (2006).

[56] C. C. McCluskey. Global stability for a class of mass action systems allowing for latency in tuberculosis. J. Math. Anal. Appl., (2007).

[57] J. MenaLorca, H. W. Hethcote. Dynamic models of infectious diseases as regulators of population sizes. J. Math. Biol., 30 (1992), 693- 716. 
[58] J. S. Muldowney. Compound matrices and ordinary di? erential equations. Rocky Mountain J. Math., 20 (1990), 857-872.

[59] C. P. Simon, J. A. Jacquez. Reproduction numbers and the stability of equilibria of SI models for heterogeneous populations. SIAM J. Appl. Math., 52 (1992), 541-576.

[60] H. Smith. Periodic orbits of competitive and cooperative systems. J. Differential Equations, (1986), 361-373.

[61] H. Smith. Monotone dynamical systems. An introduction to the theory of competitive and cooperative systems. AMS, Providence, R.I., 1995.

[62] R. Smith. Some applications of Hausdorff dimension inequalities for ordinary differential equations. Proc. Roy. Soc. Edinburgh, 104 (1986), 235-259.

[63] Y. Takeuchi, N. Adachi. The existence of globally stable equilibria of ecosystems of the generalized volterra type. J. Math. Biol., (1980), 401- 415.

[64] H. R. Thieme. Global asymptotic stability in epidemic models. In: Equadiff 82, Proc. int. Conf., Wurzburg 1982, No. 1017 in Lectures Notes in Biomath., Springer Verlag, (1983), 608-615.

[65] H. R. Thieme. Local stability in epidemic models for heterogeneous populations. In: Mathematics in biology and medicine, Proc. Int. Conf., Bari/Italy 1983, Lect. Notes Biomath. 57, Springer Verlag, (1985), 185-189.

[66] H. R. Thieme. Mathematics in population biology. Princeton Series in Theoretical and Computational Biology. Princeton University Press, Princeton, NJ, 2003.

[67] P. van den Driessche, J. Watmough. Reproduction numbers and subthreshold endemic equilibria for compartmental models of disease transmission. Math. Biosci., (2002), 29-48.

[68] R. Varga. Factorization and normalized iterative methods in Boundary problems in differential equations. R. Langer, ed., university of Wisconsin Press, (1960), 121-142.

[69] R. Varga. Matrix iterative analysis. Prentice Hall, 1962. 




Figure 1: The two susceptible classes and two infectious classes model 